\title{
Implementation of Gas Detection System using Unmanned Moving Vehicle
}

\author{
Chinchu V Joy ${ }^{1}$, Gnana Sheela $\mathrm{K}^{2}$, Manu Augustine ${ }^{3}$ \\ P.G Student, Dept of ECE, TIST, Ernakulam, Kerala ${ }^{1}$ \\ Faculty, Dept of ECE, TIST, Ernakulam, Kerala ${ }^{2}$ \\ Arvin Technologies, Ernakulam, Kerala ${ }^{3}$
}

\begin{abstract}
Unmanned moving vehicles are nowadays largely used in environment monitoring system. In order to identify the leakage of gas in a housing area or an industry or in an agricultural area, it can be easily monitored and detected by the sensors that are embedded on a moving vehicle. A remote controlled vehicle is used in the proposed system. With help of camera attached to this the area where hazardous gas leakage can be identified. In case of emergency like fire explosion in some other area the vehicle can be manually moved to that location. The information about the gas leakage is transferred through ZIGBEE. GPS is used to trace the location where leakage has happened. The leakage of harmful gas in agricultural area, housing area and industrial area can be detected more accurately.
\end{abstract}

Keywords: Unmanned moving vehicle, ZIGBEE, GPS, Gas Detection.

\section{INTRODUCTION}

Unmanned moving vehicles are widely used for data collection and analysis. Data collected by these vehicles will be more accurate than a human operator collecting data. A mobile robot attached with sensors and camera can continuously monitor the environment conditions where it gives correct details. It will provide a safe situation because it is not possible for a human operator to monitor the area where gas leakage has happened. It creates danger for human and also the environment.

The life on earth exists because of air and water. What happens if both are polluted? This is the situation in most of the urban areas. Air and water are being polluted at a rapid rate due to the excess use of chemicals, fertilizers, emissions of gases from vehicle, leakage of gas from household etc. Gas emissions create respiratory illness and also environmental problems like acid rain, ozone layer depletion etc. Gases are volatile compounds which are colourless, odourless and tasteless. So when these compounds spread in air it will be difficult to identify with human naked eye.

At present several methods are available which detect the gas leakage. But the drawback of these systems is it uses a static network of sensors. By using this static network the correct source of leakage cannot be identified. The gas as soon as it leaked it will spread very fast in air. Thus a proper device should be required to detect the leakage.

In this paper, an embedded electronic platform which can instrument a moving vehicle with chemical sensing system for gas leakage detection and environment monitoring. This system consists of an ARM microcontroller which is 64 bit. GPS used for detecting the location of gas leakage. ZIGBEE is used for transmit and receive data. Five MQ series sensors are attached to the system which is used for detecting LPG, methane, ammonia, carbon monoxide and humidity. A camera is attached to this system for monitoring the area where there is possibility for the gas leakage. The vehicle moves through a predefined path. This device works in two modes firstly it works in predefined path. Secondly it move by manually i.e. as instructions given by the controller.

The paper is structured as follows: Related works are depicted in section II. The implemented control system is described in section III. The hardware description is given in section IV. The conclusion is presented in section V.

\section{RELATED WORKS}

Unmanned moving vehicles have wide range of application for data collection and sensing. Drone is commonly used for collecting data in military applications. But there is a disadvantage with the use of drone for gas sensing. The drone has sharp blade so sometimes it will be difficult to control that. Also energy autonomy of the drone will be higher.

During the last decades, different types of sensor networks were used. The data collected with this network were not accurate and specific. It is not possible to detect the area of gas leakage by human because during the time of leakage the gas will spread in wide range. The molecules of gas will mix with the air within short time. So the previous static network of sensors cannot be used to properly detect the gas leakage.

A technology developed by N.M. Tamarin et.al (2012) discussed about Simultaneous Localization and Mapping Based Real-Time Inter-Row Tree Tracking Technique for Unmanned Aerial Vehicle. This introduces a review of previous work done using unmanned autonomic vehicles in the agricultural field, particularly in the real-time inter- 
Vol. 4, Issue 2, February 2017

row tracking and localization techniques. A new method was introduced in row detection techniques based on simultaneous localization and mapping .The inter-row tracking technique is different from other available approaches done for detection and navigation. This technique is purposely used for small scaled unmanned aerial vehicle where substantial devices or equipments is not possible due to its enormous weight and size.

Vision-based, laser-based and stereo vision-based techniques are found to be an accurate row detection technique. The autonomic vehicles and robots to the admissible way points, the GPS receiver allow the system for precise navigation and localization in the previous implementation. Unfortunately, it becomes impractical in applications where there are no GPS signals or receptions in the canopied environment like in the agriculture fields e.g. palm oil, rubber and papaya. Thus, the proposed technique applies an uncomplicated algorithmic sensory measurement at the two visible landmarks, they are trees, and uses them to predict the admissible way points for UAV navigation without carrying the burden of the substantial computers, monolithic computations and distortion of the GPS signals.

M. Rossi et.al (2013) proposed a novel method to measure natural gas presence in air, using commercial off the self MOX gas sensors in wireless sensor networks. This technique were used because it reduces the power consumed by the catalytic sensors of a factor $10 \mathrm{xs}$, by performing an analysis on a reduced sampled period and thus it extends the autonomy of battery powered system. The information about the gas concentration is obtained from the sensor transient response using a discrete cosine transforms (DCT) analysis. This helps to discriminate between clean air and hazardous situations. There is two different duty cycle rates. They are characterized and simulated to demonstrate that it is possible to achieve up to 75 weeks of autonomy using commercial wireless sensor nodes, which are powered by two AA batteries.

The commercial sensors such as as-mlk MOX $\mathrm{CH} 4$ sensors from Applied Sensors are used. The proposed strategy was evaluated whether to know the gas concentration. The work demonstrates an innovative strategy for chemo resistive MOX gas sensors which achieves ultra low power performance in environmental monitoring applications. This method was intended for duty-cycled indoor air quality monitoring and volatile chemical sensing where dangerous situations are unlikely to happen. Thus a remarkable energy saving in real time applications was achieved where sensors are always switched on. As a result of this 10 times the energy required for the use of the MOX sensors is reduced. For the qualitative and immediate evaluation of the air quality needs a DCT of the transient sensor response $(512 \mathrm{~ms}$ switch on time against the standard $5 \mathrm{~s}$ to achieve a quantitative measure).Thus the characterization of a commercial MOX sensor which was targeted to natural gas measurement has been performed against a range of relative humidity conditions.
During the year 2014 M. Rossi et.al developed Gas Sensing System on using UAV for Localization of Gas leakage. Chemical concentration of gas and leakage recognition will go dangerous in environment. The use of these kinds of Unmanned Aerial Vehicles (UAVs) for the measuring of spatially distributed gas concentration is of great importance because it gives a Simultaneous Localization and Mapping (SLAM) of the volatiles. The design of integrated sensing instruments that focus on the optimization of crucial features as weight, dimension and energy autonomy, as important as selectivity and sensitivity of sensors on board UAVs.

This proposed Gas Sensing System (GSS) is a fully autonomous board based on a 32bit MCU with 30min autonomy (on its own battery). This is used for data storing, wireless connectivity for real-time feedback and embeds a custom micro-machined MOX (Metal Oxide) sensor. This system can be mounted on any UAV because of its smaller [1] dimensions and light weight. Experiments demonstrate that the sensing performance is not impaired by the air. The Gas Sensing System (GSS) aims at joins: (i) drone mapping and (ii) gas concentration analysis.

\section{PROPOSED SYSTEM}

In the proposed system is a device that can detect the leakage of gas in the housing area is considered. This system is a mobile robot which is equipped with embedded system i.e, having a group of sensors and a camera. The different types of sensors that are attached are CO, LPG, methane, ammonia and humidity sensors. These sensors can sense the concentration of gas spread in the atmosphere. If this is beyond the threshold value then it will be indicated as pollution. The ADC value sensed by the sensors will be displayed in PPM concentration on the LCD. The robot can work in two modes. In first mode it moves through the predefined path ie it continuously monitors the housing area. The person who is sitting in the control room can monitor that area. The location is traced using GPS. The data transmission and reception is done by ZIGBEE module.

During the second mode, the robot can be moved to some other location to identify the leakage or fire explosion. By giving command through ZIGBEE module is used for the movement of system.

\section{HARDWARE DESCRIPTION}

The system is implemented using ARM Microcontroller. The ARM microcontroller is interfaced with $\mathrm{CO}$ sensor, LPG sensor, methane sensor, ammonia sensor and humidity sensor.An LCD is used to display the values of harmful gases sensed by the sensors.

\section{A. Zig Bee module}

Zig Bee is IEEE 802.15.4 based standard which is used for high-level communication protocols. They are used with small and low-power digital radios, such as for home 
Vol. 4, Issue 2, February 2017

automation, medical device data collection. Zigbee is used for the transmission and reception purposes. The robot will be controlled through zigbee module.

\section{B.GPS}

GPS stands for Global Positioning System. This is a space based radio navigation system. It is used to trace the correct location. By using GPS, the location where gas leakage has happened.

\section{LPG sensor}

LPG sensor is used to detect the presence of harmful gas which is released from the houses. MQ series is used for the detection of gases.

\section{Hardware setup}

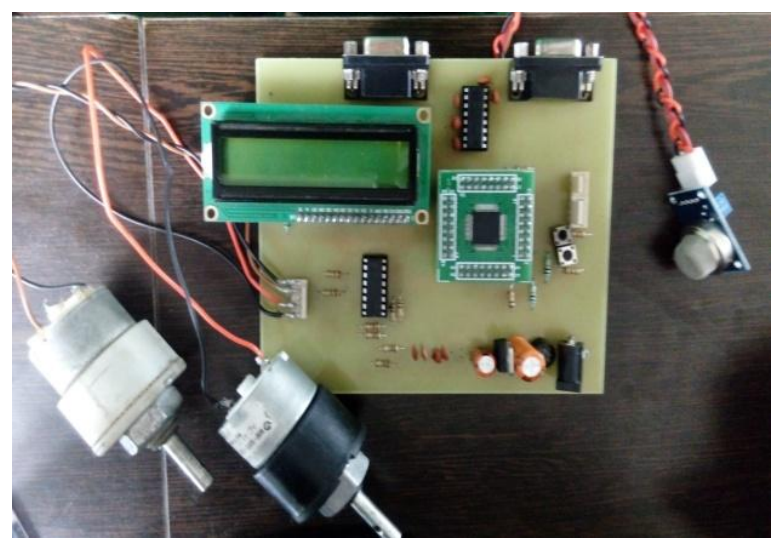

Fig1. Hardware implementation of proposed system

Fig1 shows the hardware implementation of the proposed system. The hardware configuration includes ARM microcontroller which is a 64 pin ic.L293D motor driver for interfacing of motors for robotic mechanism. For the movement of motor current should flow in bi direction. So for that movement of current L293D is used because it has $\mathrm{H}$ Bridge in it. MAX232 is used for making RS232 and TTL logic compatible. USART is used for transmission and reception proposes. LPG sensor belongs to MQ series used for gas detection.

\section{CONCLUSION}

Mobile robot with sensors can identify the leakage of gases. This robot will continuously monitor the housing area. The concentration of gas above the threshold level will be considered as pollution. The air pollution is a serious issue to our environment so this system helps to identify the leakage of gas in air. The mobile robot cannot complete the path if any traffic occurs in its path. It moves in a predefined path of proposed system. Thus a technique to overcome this is under consideration.

\section{REFRENCES}

[1] V. Gallego, M. Rossi, and D. Brunelli, "Unmanned aerial gas leakage localization and mapping using micro drones," in Proc. IEEE Sensors Appl. Symp. (SAS), Apr. 2015, pp. 1-6.
[2] M. Rossi, D. Brunelli, A. Adami, L. Lorenzelli, F. Menna, and F. Remondino, "Gas-drone: Portable gas sensing system on UAVs for gas leakage localization," in Proc. IEEE SENSORS, Nov. 2014, pp. 1431-1434.

[3] J. K. Hart and K. Martinez, "Environmental sensor networks: A revolution in the earth system science?" Earth-Sci. Rev., vol. 78,nos. 3-4, pp. 177-191, 2006.

[4] E. Santamaria, F. Segor, and I. Tchouchenkov, "Rapid aerial mapping with multiple heterogeneous unmanned vehicles," Int. J. Adv.Syst. Meas., vol. 6, nos. 3-4, pp. 384-393, 2013

[5] F. Nex and F. Remondino, "UAV for 3D mapping applications: A review," Appl. Geomatics, vol. 6, no. 1, pp. 1-15, 2014.

[6] P. P. Neumann, S. Asadi, A. J. Lilienthal, M. Bartholmai, and J. H. Schiller, "Autonomous gas-sensitive microdrone: Wind vector estimation and gas distribution mapping," IEEE Robot. Autom. Mag., vol. 19, no. 1, pp. 50-61, Mar. 2012.

[7] D. Caltabiano, G. Muscato, A. Orlando, C. Federico, G. Giudice, and S. Guerrieri, "Architecture of a UAV for volcanic gas sampling," in Proc. 10th IEEE Conf. Emerg. Technol. Factory Autom. (ETFA), vol. 1. Sep. 2005, pp. 739-744. 